\title{
Visual Improvement in Patients with Far-Advanced Glaucoma
}

\author{
George L Spaeth, Parul Ichhpujani \\ Anna V Goldberg Glaucoma Service, Wills Eye Institute, 840 Walnut Street, Philadelphia, PA, USA
}

\section{INTRODUCTION}

To date, there is no way to predict accurately the exact lower level of IOP at which no neuronal loss will occur in patients with glaucoma. ${ }^{1-3}$ We report here three individuals with faradvanced glaucoma unquestionably deteriorating, but who stopped getting worse when IOP was lowered to a level where vision not only stopped getting worse, but improved strikingly. We pose the questions, "How can the best tolerated level of intraocular pressure, from the point of view of the retinal ganglion cells, be established? Is improvement in function an indication that IOP has been substantially reduced? When patients with glaucoma are getting worse due to progressive visual loss, should pressure lowering be attempted when IOP is in a range usually considered tolerable (10-12 mm Hg)?”

\section{CASE REPORTS}

\section{Case 1}

A sixty-three years old organist of European extraction (Mr. S) had been followed for severe primary open-angle glaucoma for around 20 years. The left eye had no light perception when first examined in our office. The right eye at that time had faradvanced glaucoma (Fig. 1), and best corrected visual acuity (BCVA) of 20/100. Over the next 20 years, despite medicinal treatment, trabeculectomy, combined cataract extraction/ trabeculectomy with mitomycin-C, and the placement of a Baerveldt glaucoma drainage device, the visual field and BCVA in the right eye progressively deteriorated to 20/200 and later to 20/400. He still could read large print and play the organ, however. Approximately one year following the last surgical procedure the IOP rose to around $15 \mathrm{~mm} \mathrm{Hg}$ despite medicinal therapy. Several weeks after noting the pressure rise, the patient called to say that he had lost all useful vision in his right eye. At the time of a new examination, the visual acuity had deteriorated to poor hand movements. Repeated testing by different examiners confirmed that there was no ability to see any size test letter at any distance. The patient could no longer read at all; he said there had been a sudden, marked change from his usual state. He started using a cane for mobility and consulted an agency for the blind. IOP was in the low to mid-teens. There was no corneal edema, no macular edema, and no other detectible cause for visual deterioration except progressive glaucoma. The visual field, which had been progressively worsening in a fashion characteristic of glaucoma and had been limited to around $5^{\circ}$ centrally using a stimulus III of the Humphrey field analyzer, was unobtainable with a Humphrey stimulus V; the central island of vision disappeared. Because the patient was desperate and the deterioration in acuity and field was definite, a further attempt to lower intraocular pressure appeared appropriate. An endoscopic cyclophotocoagulation (ECP) was performed. Following this the IOP fell to around $6 \mathrm{~mm} \mathrm{Hg}$. Within one week, the patient noted what he called an amazing improvement. Two weeks later he commented that he was seeing better than he had "for years." He no longer used his cane, he was able to read again, and his brother, who was his caregiver, commented that the patient was now completely able to care for himself. BCVA had improved to 20/200. His visual field was still "end stage," but a central island was easily plotted.

Other than the change in IOP, there was no apparent explanation for the change in visual acuity. The refractive error was unchanged, the corneal thickness remained the same, and there were no noticeable alterations in the retina.

Improvement lasted for approximately one month, at which time the IOP had risen to $8 \mathrm{~mm} \mathrm{Hg}$ and visual acuity fell to hand movements. However, the patient continued to comment on how much better he was able to "see" and care for himself than he was prior to the ECP. He no longer needed a cane for mobility, and he continued to care for himself without assistance.

\section{Case 2}

$\mathrm{Mr} \mathrm{H}$, a 54-year-old highly-myopic individual had a complicated eye history and a positive family history of glaucoma. He developed pigmentary glaucoma in his 20's, the control of which 


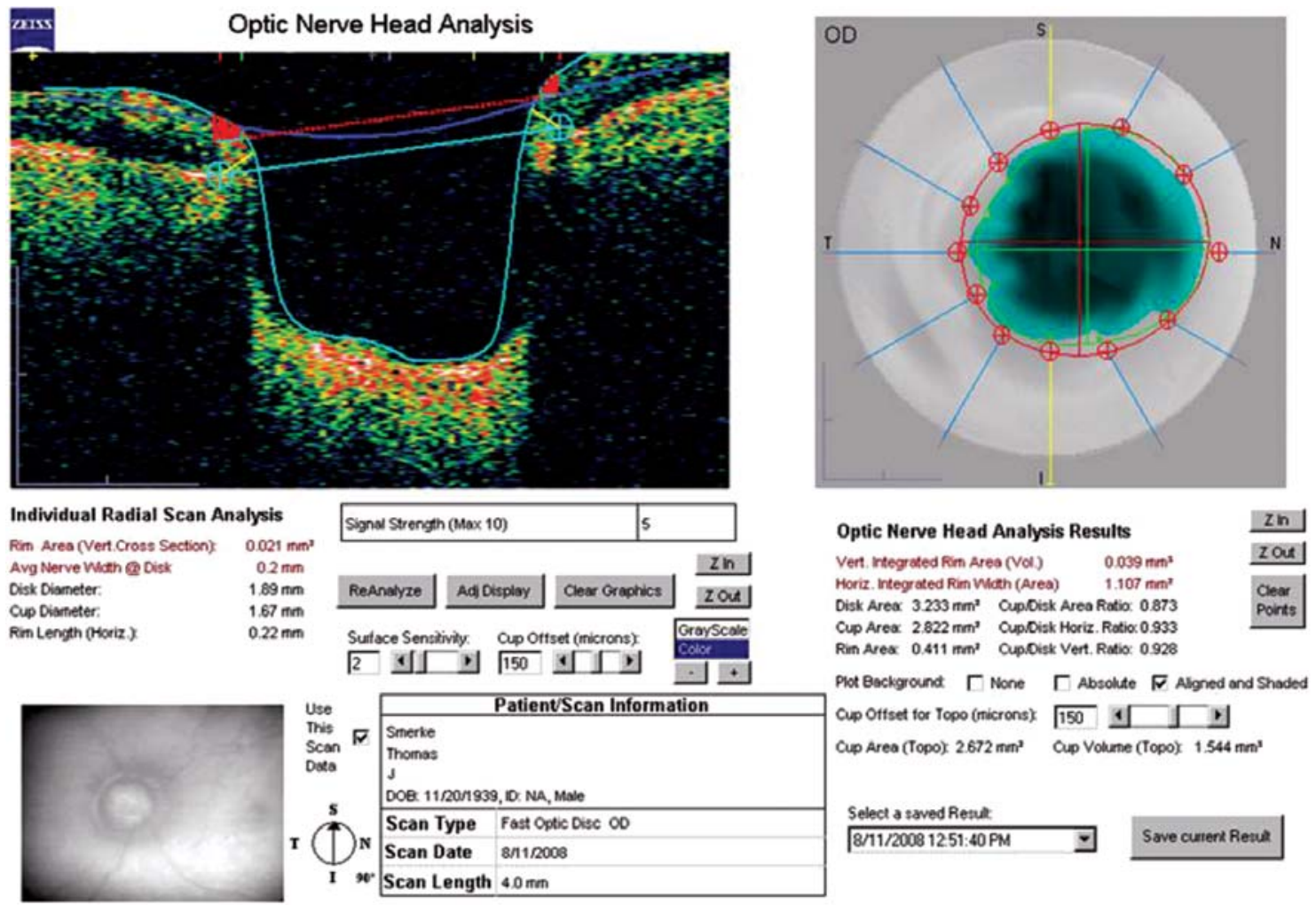

Fig. 1: Fast optic nerve head scan of right eye of case 1, showing near total cupping

was difficult. Both eyes developed retinal detachments which were successfully repaired with scleral buckling. Despite trabeculectomies and tube shunt procedures, his optic nerves became progressively damaged (Fig. 2) and his vision field deteriorated (Both eyes: Mean defect -21). His wife commented on his serious decrease in ability to function. His IOP rose from the low teens into the mid- and upper-teens. Because of this continuing escalation of IOP despite topical and systemic medications, and because of reducing BCVA to 20/100 in the right eye and 20/200 in the left eye, he was treated with an ECP. Following this his IOP fell to $5 \mathrm{~mm} \mathrm{Hg}$ and he developed moderate intraocular inflammation. Within one week after the procedure, he noted a dramatic improvement in his vision. In his words, "I could see every pebble in the driveway. My vision hadn't been that good for years!” BCVA was 20/50 in the right eye and 20/ 200 in the left eye. Four months following the ECP, BCVA was 20/50 in the right eye and 20/60 in the left eye. He continued to comment on the remarkable improvement in his ability to get around.

\section{Case 3}

Dr G, a brilliant mathematician involved in the development of the computer, came to our practice after having had trabeculectomies performed in both eyes for average-pressure glaucoma. His visual acuity was 20/20, but he had marked loss of the visual field (superior field more than inferior field) in both eyes with a Humphrey Stimulus III (Fig. 3). The IOP was consistently $11 \mathrm{~mm} \mathrm{Hg}$ in both eyes for ten years, due to the excellent filtering procedures which had been performed prior to coming to our office. During that period of time visual fields, which were repeated at least yearly, were always amazingly unchanged. At one visit, however, he mentioned that his color vision in his right eye had deteriorated markedly and he could no longer accurately identify colors. His IOP had risen to $13 \mathrm{~mm} \mathrm{Hg}$ in the right eye but remained at 11 in the left. Acuity was still 20/20 in each eye. The visual field examination showed three spots in the right eye which had deteriorated $7 \mathrm{~dB}$. The left eye was unchanged from before. IOP was $13 \mathrm{~mm} \mathrm{Hg}$. in the right eye and 11 in the left eye. An argon laser trabeculoplasty was performed on the right eye, following which the IOP fell to 10 $\mathrm{mm} \mathrm{Hg}$ in the right eye and continued to be $11 \mathrm{~mm} \mathrm{Hg}$ in the left eye. He noted complete recovery of his color vision and the three reduced points in the visual field returned to normal.

\section{DISCUSSION}

All three of the individuals reported here had long-standing glaucoma, to which they had adjusted well. All had been followed for over ten years and reliable determinations of acuity and field performed. The three individuals noted what they considered unmistakable deterioration in their vision, which 


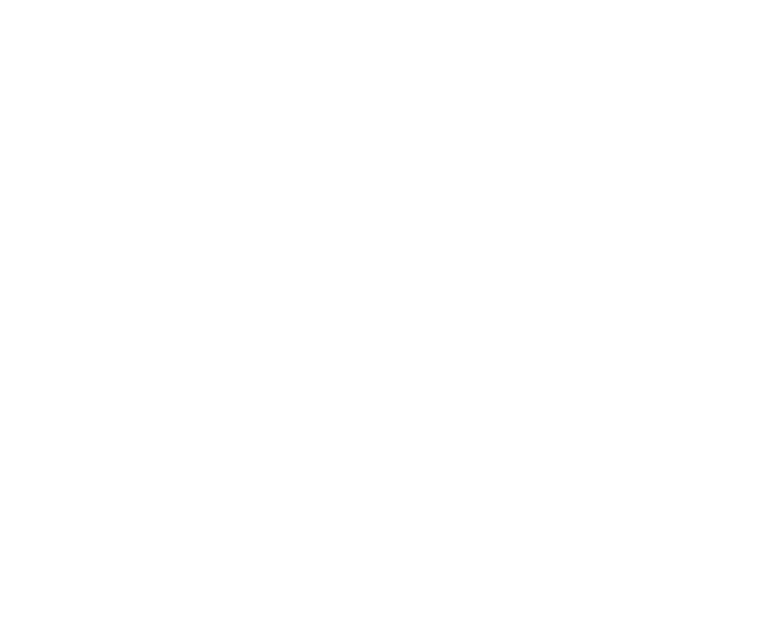

Fig. 2: Monophotograph of right optic disk of case 2

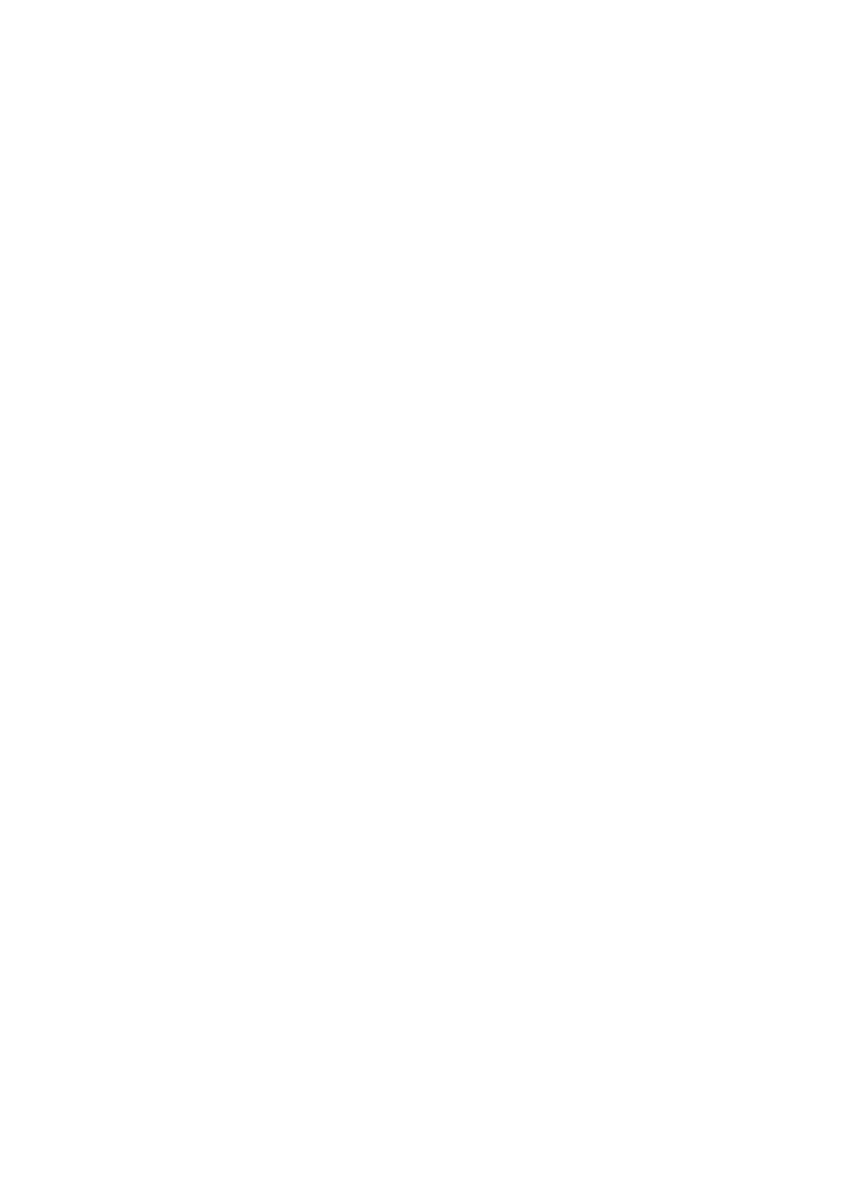

Fig. 3: Serial central 10-2 visual fields of right eye of case 3 was supported by clinical findings of worsening acuity and visual field in the first two patients, and worsening field in the third patient. All three cases were pseudophakic. None had detectible change in the macula, or other cause for reducing vision. However, the most dramatic aspect of their condition was their clinical symptomotology. In all three cases following marked lowering of IOP after ECP, all had a dramatic improvement in their vision and their ability to function. The changes in visual ability led to marked improvement in their ability to care for themselves and to function more generally, as noted not only by the patient, but by the patients' partners. While the changes in Case 3 were far more subtle than either of the first two cases, they were just as believable. Case 3 was followed-up for 7 years without any further deterioration, with an IOP ranging between 9-11mm Hg and stability of visual field.

\section{CONCLUSION}

This case series suggests that IOP lowering not only can lead to better clinical outcome, but also to improvement in patients' functioning in day to day activities and psychological profile.

\section{REFERENCES}

1. Eddy DM, Sanders LE, Eddy JF. The value of screening for glaucoma with tonometry. Surv Ophthalmol 1983;28:194-205.

2. Wormald R. Treatment of raised intraocular pressure and prevention of glaucoma. BMJ 2003;326:723-24.

3. Mackenzie P, Cioffi G. How does lowering of intraocular pressure protect the optic nerve? Surv Ophthalmol 2008;53(Suppl 1):S3943.

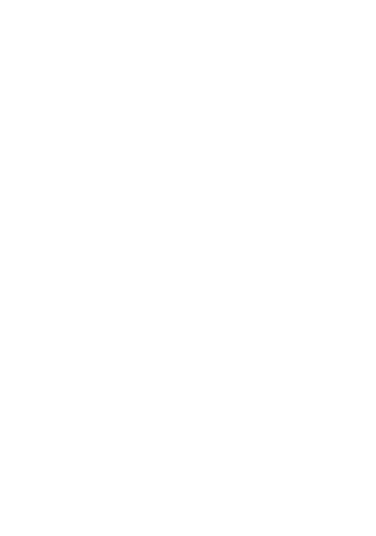

George L Spaeth (gspaeth@willseye.org) 